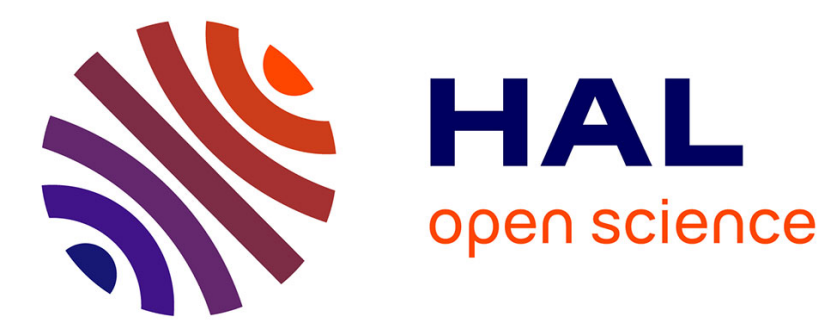

\title{
La pertinence de la politique rurale à l'aune des théories de la justice
}

Y. Schaeffer, Francis Aubert

\section{To cite this version:}

Y. Schaeffer, Francis Aubert. La pertinence de la politique rurale à l'aune des théories de la justice. Revue d'Etudes en Agriculture et Environnement - Review of agricultural and environmental studies, 2010, 91 (1), p. 73 - p. 101 . hal-00583623

\section{HAL Id: hal-00583623 https://hal.science/hal-00583623}

Submitted on 6 Apr 2011

HAL is a multi-disciplinary open access archive for the deposit and dissemination of scientific research documents, whether they are published or not. The documents may come from teaching and research institutions in France or abroad, or from public or private research centers.
L'archive ouverte pluridisciplinaire HAL, est destinée au dépôt et à la diffusion de documents scientifiques de niveau recherche, publiés ou non, émanant des établissements d'enseignement et de recherche français ou étrangers, des laboratoires publics ou privés. 


\title{
La pertinence de la politique rurale à l'aune des théories de la justice
}

\author{
Yves SCHAEFFER*, Francis AUBERT** \\ * Auteur correspondant : CEMAGREF, UR DTGR, 2 rue de la Papeterie, \\ BP 76, F-38402 St Martin d'Hères et AgroSup Dijon, UMR-1041 CESAER, \\ université de Bourgogne, France \\ e-mail : yves.schaeffer@cemagref.fr \\ ** AgroSup, UMR-1041 CESAER, Dijon, France
}

\begin{abstract}
Résumé - Comment évaluer la pertinence de la politique rurale française? S'il s'agit de savoir si les mesures mises en œuvre permettront d'atteindre les objectifs poursuivis, la démarche est assez bien balisée. En revanche, s'il s'agit de porter un jugement sur les objectifs visés, une difficulté majeure apparaît : le référentiel de cette évaluation est indéfini. Dans cet article, nous proposons de faire appel à la philosophie morale et politique pour le constituer : les objectifs de la politique rurale sont pertinents s'ils dérivent d'une conception de la justice dont la pertinence philosophique est établie. Selon cette perspective, la pertinence des orientations actuelles de la politique rurale est incertaine, car leurs fondements philosophiques sont fragiles et contestables. Au-delà de l'analyse d'une politique particulière, nous souhaitons souligner l'importance de la question de l'adoption par l'Etat d'une conception de la justice sur laquelle fonder son action.
\end{abstract}

Mots-clés : politique rurale, évaluation, pertinence, justice sociale, Rawls

The relevance of the French rural policy by the yardstick of the theories of justice

Summary - How to evaluate the relevance of the French rural policy? When the question is to what extent the objectives of the policy are reached owing to public interventions, the method is relatively well known. However, when the question is to what extent these objectives are relevant, an important problem is faced: the referential of this evaluation is indefinite. In this article, we propose to define this referential on the basis of moral and political philosophy: The objectives are relevant if they are derived from a conception of justice that is itself relevant. From this viewpoint, the relevance of the current orientations of the rural policy is dubious, since they rest on weak and questionable philosophical foundations. Beyond this analysis, we want to stress on the importance for the State to adopt a clear conception of justice, so as to firmly found its action.

Keywords: rural policy, evaluation, relevance, social justice, Rawls

Descripteurs JEL : D63, R58

Nous tenons à remercier les deux rapporteurs anonymes pour leurs commentaires avisés. 


\section{Introduction}

Le plan stratégique national de développement rural 2007-2013 présente de manière unifiée «la politique nationale globale de développement rural» (Ministère de l'Agriculture et de la Pêche, 2007). Il en expose les orientations générales - objectifs et modalités d'action retenus par l'Etat - et recense l'ensemble des instruments nationaux assurant leur mise en œuvre. Au niveau des instruments, l'étendue et la complexité de cette politique sont manifestes. A côté du fonds européen agricole de développement de l'espace rural, trois types d'instruments sont identifiés : les lois d'orientation, les lois et les plans d'action. Les lois d'orientation sont au nombre de trois et intéressent l'agriculture, le développement des territoires ruraux et la forêt. Huit lois sont dénombrées, portant sur la montagne, les paysages, l'eau, les milieux aquatiques, les habitats et les oiseaux, l'initiative économique et la chasse. Plus de dix plans d'action sont en cours ${ }^{1}$. Au niveau des modalités d'action, en revanche, une priorité est clairement énoncée : tirer parti de la diversité des territoires et du potentiel de leurs acteurs via l'accompagnement des projets locaux d'aide à la création de richesses. Au niveau des objectifs, enfin, une certaine complexité est à nouveau de mise, faisant intervenir différents échelons géographiques: la finalité globale est la valorisation économique, sociale et environnementale des territoires ruraux et, dans le même temps, il s'agit d'assurer à l'échelle nationale la croissance économique et la création d'emploi, la cohésion sociale et territoriale, la justice sociale et l'équité territoriale, la préservation de la diversité des territoires et la durabilité environnementale.

Quelle est la pertinence de cette politique? Une évaluation d'ensemble requiert de porter un jugement sur ses objectifs, ses modalités d'action et chacun de ses instruments de mise en œuvre. Nous n'avons évidemment pas la prétention d'aborder ici tous ces points. La question de savoir si les instruments et les modalités sont les plus appropriés pour assurer l'atteinte des objectifs poursuivis est bien connue et constitue le cœur de la littérature d'évaluation. En revanche, la question de savoir si les objectifs retenus sont pertinents soulève une difficulté importante: le référentiel de cette évaluation n'est en rien évident. Le référentiel proposé usuellement par le corpus de l'évaluation de politique est celui des «problèmes et (des). besoins perçus par la population, les décideurs publics et l'évaluateur» (Perrin et al., 2003, p. 121). Dans son ouvrage de référence sur les pratiques d'évaluation, la Commission européenne définit la pertinence comme étant "l'adéquation entre les objectifs explicites de l'intervention et les problèmes socioéconomiques» (Commission européenne, 1999, p. 65). A contrario, nous proposons de faire appel à la philosophie morale et politique pour constituer ce référentiel d'évaluation. Selon cette perspective, les objectifs de la politique rurale sont

\footnotetext{
${ }_{1}^{1}$ Ils concernent l'industrie agroalimentaire, l'agriculture biologique, la biodiversité, les biocarburants, les biocombustibles, les bioproduits et biomatériaux, les risques liés aux pesticides, la forêt, les territoires ruraux, les pôles d'excellence rurale et les pollutions d'origine agricole. Cet inventaire est de plus relativement restrictif. L'instance d'évaluation de la politique rurale, mise en place par le Commissariat général du plan en 2001, avait recensé les dispositifs mis en œuvre durant la décennie 90 (Perrin et al., 2003) : plus de soixante dispositifs publics de développement rural avaient été identifiés, touchant à tous les domaines de la vie économique et sociale et mobilisant des montants annuels de plus de deux milliards d'euros.
} 
pertinents s'ils dérivent d'une conception de la justice dont la pertinence philosophique est établie.

Dans la partie suivante, nous présentons les orientations historiques et actuelles de la politique rurale et nous développons le problème posé par l'évaluation de ses objectifs. La troisième partie expose quelques conceptions de la justice que nous mobilisons dans la suite de notre discussion. Deux démarches sont alors entreprises dans la quatrième partie. D'une part, nous tentons d'identifier les fondements philosophiques implicites des orientations actuelles de la politique rurale française et, d'autre part, nous imaginons ce que pourraient être des orientations fondées sur d'autres conceptions de la justice. Nous revenons finalement, dans la dernière partie, à la question de l'évaluation de la pertinence des objectifs de la politique rurale, en nous appuyant sur l'exposé du débat philosophique sur la justice.

\section{La politique rurale française}

Nous concevons les orientations de la politique rurale comme un ensemble d'objectifs dont l'Etat souhaite favoriser la réalisation (par exemple, améliorer les conditions de vie de la population rurale), associé à un ensemble de modalités d'action retenues à cette fin (par exemple, moderniser les exploitations agricoles) ${ }^{2}$. Des instruments publics les mettent en œuvre (par exemple, un dispositif de prêts bonifiés). Dans cette partie, nous présentons une brève histoire de la politique rurale française depuis la fin de la Seconde Guerre mondiale, puis nous précisons les orientations actuellement en vigueur. Le problème que soulève l'évaluation de leur pertinence, lorsque celle-ci se concentre sur le niveau des objectifs, est discuté dans une dernière sous-section.

\subsection{Quelques éléments d'histoire de la politique rurale}

Une périodisation historique simple aboutit à distinguer trois temps depuis la fin de la Seconde Guerre mondiale, mettant en exergue des orientations principales que nous qualifions par leurs modalités d'action emblématiques: "l'aménagement des campagnes et la modernisation de l'agriculture » tout d'abord, puis «le rééquilibrage d'ensemble de la géographie nationale des activités et des services», et enfin «le soutien aux projets de territoires ».

\subsubsection{L'aménagement des campagnes et la modernisation de l'agriculture}

Le premier temps couvre les années 1950 et le début des années 1960. Dans un contexte de reconstruction et de modernisation du pays, la politique rurale a pour objectifs d'améliorer les conditions de vie des ruraux et de contribuer à établir les

\footnotetext{
${ }^{2}$ La distinction entre ce que nous appelons ici «objectifs» et «modalités d'action » n'est pas toujours évidente à établir. Un simple changement de terminologie permet de s'en rendre compte : les secondes pourraient tout aussi bien être nommées "objectifs opérationnels », et les premiers «objectifs finaux » ou « finalités ». Il s'agit, en fait, de différents maillons d'une chaîne d'objectifs : les seconds se trouvant au service des premiers. Nous réservons la dénomination d'objectifs à ceux qui se trouvent tout au début de la chaîne, et appelons modalités les autres.
} 
conditions du développement économique national. L’aménagement des campagnes constitue une première modalité : des fonds nationaux financent la généralisation de l'accès à l'eau potable, à l'assainissement et à l'électricité (fonds national de développement des adductions d'eau, fonds d'amortissement des charges d'électrification, etc.); des sociétés d'économie mixte (Compagnie nationale d'aménagement du Bas-Rhône et du Languedoc, Société de mise en valeur de l'Auvergne et du Limousin, etc.) procèdent aux grands travaux concernant la maîtrise de l'eau (drainage, irrigation, contrôle des flux, etc.) et le désenclavement des territoires (voies de communication, défrichement, etc.). Au début des années 1960, la modernisation de l'agriculture en devient la modalité principale. Celle-ci assure une amélioration des conditions de vie des agriculteurs, elle-même posée comme une exigence d'équité: "L'agriculteur moderne a droit à une meilleure vie » (présentation de la loi d'orientation agricole au Parlement, 1960) ; "Assurer un niveau de vie équitable à la population agricole » (Traité de Rome, 1957, art. 39). Plus largement, le développement agricole est considéré comme le principal moteur du développement de l'économie rurale et le meilleur moyen d'améliorer les conditions de vie de l'ensemble des ruraux. La politique rurale admet enfin un troisième objectif, marginal, de préservation des milieux naturels. Il se traduit par la création de parcs nationaux dès 1963 (Parc de la Vanoise, de Port-Cros, etc.).

\subsubsection{Le rééquilibrage d'ensemble de la géographie nationale des activités et des services}

Le deuxième temps s'étend du milieu des années 1960 au milieu des années 1980. Il s'inscrit dans un contexte de généralisation progressive des normes urbaines sur le plan des modes de vie, de consommation et de travail. Les orientations de la politique rurale s'identifient à celles de la politique d'aménagement du territoire, dont la finalité principale est de contribuer à une meilleure répartition des richesses à l'échelle nationale : «le temps est révolu où la prospérité des uns pouvait s'accommoder de la misère des autres» (présentation du décret de création de la délégation à l'aménagement du territoire et à l'action régionale - DATAR - à l'Assemblée nationale, 1963); "l'ambition de l'aménagement du territoire est de faire que la richesse nationale soit mieux répartie, que l'expansion profite autant au citadin qu'au rural, à l'ouvrier qu'au paysan, aux babitants d'une région qu'à ceux d'une autre » (Guichard, 1965, p. 245). Sur le plan des modalités, il s'agit de rééquilibrer la géographie économique nationale au profit des zones rurales et d'égaliser autant que possible les conditions d'accès à une large gamme de services entre territoires ruraux et urbains (télécommunication, santé, etc.). Les dispositifs d'aide zonés constituent les instruments par excellence pour agir sur la géographie économique, par la compensation des «handicaps » des zones les plus «en retard» au regard des standards nationaux de développement. En 1967, des commissaires à la rénovation rurale relevant directement de la DATAR sont mis en place au niveau des régions et sont chargés d'enrayer la dévitalisation des zones rurales. La décentralisation industrielle est soutenue par l'emblématique Prime d'aménagement du territoire (1982), d'abord réservée aux activités industrielles, puis étendue aux activités tertiaires. En agriculture, des zones de handicaps sont définies sur la base des caractéristiques topologiques et pédoclimatiques des territoires et des aides spécifiques sont délivrées aux exploitations qui s'y trouvent localisées (zones «de montagne » définies en 1974, zones « agricoles défavorisées » en 1976). Pour ce qui a trait à l'accès 
aux services publics locaux, les villages sont incités à compléter leur offre grâce à des transferts dont les modalités assurent un certain degré de péréquation des dotations par habitant (dotation globale de fonctionnement créée en 1979 incluant des critères géographiques). Les questions de protection de la nature restent dans l'ensemble traitées à part, mais leur prise en compte se renforce progressivement (réserves naturelles d'intérêt général instituées en 1976, zones naturelles d'intérêt écologique, faunistique et floristique en 1983, directive Oiseaux de 1979).

\subsubsection{Le soutien aux projets de territoires}

Le dernier temps se déroule de la fin des années 1980 à aujourd'hui, dans un contexte marqué par la décentralisation politique et par le regain démographique de l'espace rural. L'existence de potentiels de développement dans les territoires ruraux est mise en évidence par les dynamiques démographiques et économiques positives observées dans un certain nombre d'entre eux. La valorisation de l'ensemble de ces potentiels de développement, dans leurs dimensions économique, social et environnementale, devient progressivement la finalité première de la politique rurale: "il s'agit de reconnaître le rôle économique, social et environnemental des espaces naturels et ruraux qui constituent un atout de développement dans l'immédiat et à long terme » (Exposé des motifs de la loi d'orientation relative à l'aménagement et au développement durable du territoire, 1999). Les modalités d'action évoluent en conséquence : «d'une logique de redistribution des ressources destinée à compenser des inégalités ou des handicaps et à soutenir des activités, à une démarche d'accompagnement des projets locaux d'aides à la création de richesses» (Exposé des motifs de la loi relative au développement des territoires ruraux, 2005). Pour le dire autrement, la priorité était d'améliorer l'allocation des ressources au niveau national dans une perspective d'équité, elle est maintenant d'améliorer l'allocation des ressources à l'échelle de chaque territoire dans une perspective supposée intégrer efficacité, équité et durabilité. La décentralisation politique favorise la création de dispositifs s'appuyant sur la participation et l'organisation des acteurs locaux: programme d'initiative communautaire LEADER en 1991, création des «Pays» à la suite des lois d'orientation relatives à l'aménagement et au développement (durable) du territoire de 1995 et de 1999, systèmes productifs locaux en 1998, puis pôles d'excellence rurale promus par la DIACT ${ }^{3}$ en 2005. Les porteurs de projets sont encouragés à prendre en compte les milieux naturels en tant que ressources territoriales à part entière, qu'il faut préserver, mais aussi valoriser. Ils sont invités en outre à considérer l'attractivité territoriale comme une variable-clé du développement local. Ces enjeux se rejoignent d'ailleurs à mesure que les aménités naturelles prennent une importance grandissante dans les choix de localisation des ménages. L'accent mis sur l'attractivité territoriale conduit également à poser d'une manière nouvelle la question de l'accès aux services (publics et, plus généralement, résidentiels): il ne s'agit plus seulement de faire correspondre la répartition des services sur le territoire national à celle des ménages, considérée comme donnée, mais d'orienter les mobilités résidentielles en ajustant les dotations territoriales en services.

3 Délégation interministérielle à l'aménagement et à la compétitivité des territoires, qui a succédé à la DATAR et redevenue DATAR en 2009. 
Cette périodisation est très schématique ${ }^{4}$. Ajoutons, tout d'abord, que l'agriculture occupe aujourd'hui encore une place importante dans la politique rurale, tant au niveau national que communautaire: "agir pour l'agriculture, c'est aussi participer au combat en faveur de l'emploi, conférer du dynamisme au monde rural et préserver nos territoires » (présentation du projet de loi d'orientation agricole à l'Assemblée nationale, 2005); "pas d'agriculture sans campagnes vivantes, pas de campagnes vivantes sans agriculture " (Déclaration de Salzbourg, 2003). De même, l'idée de rééquilibrer la géographie des activités et des services n'a pas disparu dans la période récente - «le débat national a mis en avant le souci de pouvoir accéder partout à une qualité d'infrastructures et de services à la mesure du développement du pays» (Exposé des motifs de la loi d'orientation relative au développement du territoire, 1995) - et le zonage demeure un instrument important de la politique rurale. La démarche de compensation assise sur un zonage est d'ailleurs quelquefois mise en œuvre de manière complémentaire à la démarche de soutien à des projets de territoire (la procédure des pôles d'excellence rurale, par exemple, vise préférentiellement les zones de revitalisation rurale). Inversement, si la politique d'aménagement du territoire des années 1960 avait essentiellement une ambition redistributive, elle affichait déjà l'intention de contribuer à la valorisation des territoires: «le but est de développer ou de chercher à développer les capacités et les aptitudes de chaque région, de telle manière que celle-ci puisse fournir à la prospérité générale sa juste contribution... (présentation du décret de création de la DATAR, 1963). Quant au développement durable, il avait fait son apparition dès 1968 avec la création des parcs naturels régionaux (Parc Scarpe-Escaut), associant des objectifs de préservation de milieux remarquables du point de vue naturel et culturel avec des objectifs de production.

\subsection{Les orientations de la politique rurale d'aujourd'hui}

Après ce cadrage historique, présentons plus en détails les orientations de la politique rurale actuellement en vigueur. Pour cela, deux documents font référence: le plan stratégique national (PSN) de développement rural 2007-2013, rédigé par le ministère de l'Agriculture et de la Pêche (MAP) et daté du 16 avril 2007, et la loi no 2005-157 du 23 février 2005 relative au développement des territoires ruraux (LDTR). Le premier a pour principal objet la définition du cadre stratégique pour la programmation du Fonds européen de développement de l'espace rural (FEADER), second pilier de la politique agricole commune. Cependant, il présente également les objectifs de " la politique nationale globale de développement rural " ${ }^{5}$, tous dispositifs confondus. Le second présente un ensemble de dispositions législatives qui s'apparente, d'après le législateur, à une loi d'orientation rurale 6 .

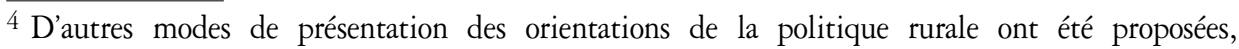
notamment, par Moulinier et Mondot (2005), par Guérin (2006), ou encore par Berriet-Solliec et al. (2009).

5 Cette dénomination est donnée par le PSN.

${ }^{6}$ Le PSN présente l'ensemble des instruments retenus par les autorités pour la mise en ouvre des objectifs de la politique nationale globale de développement rural : lois d'orientations, lois et plans d'action. La LDTR est classée parmi les premières, avec la loi d'orientation agricole et la loi d'orientation forestière.
} 


\subsubsection{Le plan stratégique national}

La politique nationale globale de développement rural, de même que la programmation 2007-2013 du FEADER, a pour finalité première de «valoriser les territoires ruraux du point de vue économique, social et environnemental» (MAP, 2007, pp. 5, 30 et 148). Sa principale modalité de mise en ouvre consiste à "(tirer) parti de la diversité et du potentiel des acteurs qui les peuplent et les animent» (ib., pp. 30 et 148) ${ }^{7}$, c'est-à-dire à impliquer les acteurs locaux et à soutenir leurs projets. Cette finalité est ensuite précisée sous la forme de trois objectifs - économique, territorial et environnemental de nature plus opérationnelle ${ }^{8}$. Le premier consiste à « développer une économie rurale compétitive et riche en emplois». Le second à "promouvoir un développement territorial pour et par les populations et les acteurs des zones rurales [et à] assurer une gestion durable des ressources naturelles et du patrimoine culturel ». Le troisième à «atteindre les objectifs de la directive cadre sur l'eau et à mettre en application la stratégie nationale pour la biodiversité ». Parallèlement, la politique rurale doit contribuer à la réalisation des finalités de trois autres politiques nationales : la politique d'emploi et de croissance, la politique d'aménagement du territoire et la stratégie nationale de développement durable (SNDD). En particulier, elle est partie prenante de la politique d'aménagement du territoire et cette dernière vise "la compétitivité économique et l'attractivité des territoires, tout en veillant à la cohésion territoriale et sociale. Elle œuvre pour cela à une répartition harmonieuse de la population et des activités, en vue d'une utilisation optimale durable de tout le territoire national». En outre, la politique rurale «épouse les objectifs [de la SNDD]: concilier le développement économique, la justice sociale et la protection de la santé et de l'environnement via la solidarité des générations et des territoires ».

\subsubsection{La loi relative au développement des territoires ruraux}

L'exposé des motifs de LDTR affiche une finalité similaire: «L'Etat, garant de la cohésion nationale et de l'équité territoriale, préserve la diversité des territoires ruraux, participe à leur valorisation économique, sociale et environnementale et définit les principes de leur développement durable». Comme dans le PSN, la création de valeur sur les territoires est placée au premier plan, la valeur n'étant pas uniquement économique, mais aussi sociale et environnementale. La préservation de la diversité des territoires ruraux est un objectif de même niveau. Il se trouvait dans le PSN de manière implicite, lorsque ce dernier enjoignait à tirer parti de la diversité. Les objectifs de cohésion nationale, d'équité territoriale et de développement durable sont ici mis en exergue ${ }^{9}$. Ils étaient présents également dans le PSN via le rattachement de la politique rurale à la politique d'aménagement du territoire et à la SNDD. A nouveau, cette finalité générale est

\footnotetext{
7 Ou encore « de la diversité de leurs fonctions » (MAP, 2007, p. 5).

8 Nous constatons à ce niveau la difficulté, évoquée précédemment, à distinguer clairement les objectifs poursuivis des modalités retenues pour les atteindre, ou autrement dit, les objectifs finaux des objectifs opérationnels.

9 Le communiqué de presse du CIADT du 3 septembre 2003, consacré au monde rural et exposant les grandes lignes du projet de LDTR, proposait la dénomination de «politique de développement rural durable ».
} 
précisée sous la forme d'une série d'objectifs de nature plus opérationnelle : « développer l'activité économique...», « renforcer leur attractivité...», «contribuer à l'équilibre entre les différents usages ", «assurer la solidarité nationale au profit des territoires les plus fragiles », « promouvoir un développement durable... », etc. On retrouve à peu de chose près la déclinaison précédente en objectifs d'ordre économique, territorial et environnemental. Celle-ci semble inspirer l'organisation des articles de la loi : le titre I concerne les dispositions relatives au développement des activités économiques; les titres II et III correspondent aux dispositions relatives aux instruments de gestion foncière et à la rénovation du patrimoine rural bâti, d'une part, et à l'accès aux services, d'autre part; le titre IV regroupe les dispositions relatives aux espaces naturels ${ }^{10}$.

Au final, sur la base des deux principaux documents récents d'orientation de la politique rurale, l'ensemble des objectifs finaux formellement assignés à cette dernière parait assez clairement identifié. Les modalités d'action et les instruments de mise en œuvre retenus mériteraient d'être développés, et il serait intéressant aussi de fournir une vue d'ensemble de la littérature d'évaluation se focalisant sur ces derniers, c'est-àdire s'attachant à répondre à la question: les modalités d'action et les instruments de mise en ouvre retenus sont-ils les plus appropriés pour atteindre les objectifs visés ? Cette question, d'une importance capitale du point de vue de la décision publique, fait l'objet de la plupart des travaux regroupés dans le corpus de l'évaluation de politique ${ }^{11}$. Nous laissons ces éléments de côté afin de centrer le propos sur notre questionnement principal, c'est-à-dire sur la question de savoir si les objectifs visés eux-mêmes sont appropriés.

\subsection{Le problème de l'évaluation de la pertinence des objectifs de la politique rurale}

Les objectifs assignés à la politique rurale sont-ils pertinents? Cette question suppose l'existence d'un référentiel, au regard duquel un jugement pourra être porté. Le référentiel proposé par le corpus de l'évaluation de politique est celui des «problèmes et (des) besoins perçus par la population, les décideurs publics et l'évaluateur» (Perrin et al., 2003, p. 121). Dans son ouvrage de référence sur les pratiques d'évaluation, la Commission européenne définit la pertinence comme étant «l'adéquation entre les objectifs explicites de l'intervention et les problèmes socio-économiques»(Commission européenne, 1999, p. 65). Pourtant, la question de la pertinence d'une politique particulière rejoint celle, plus générale, du rôle des institutions de base de la société, à laquelle la philosophie morale

\footnotetext{
${ }^{10}$ La LDTR contient également des dispositions spécifiques à des espaces particuliers, montagne et outre-mer (titres VI et VII) et à certains établissements publics (titre VI).

11 Des travaux d'analyse économique de la politique rurale permettent d'apprécier théoriquement la pertinence de modalités ou d'instruments de mise en œuvre, soit en référence à des hypothèses qualitatives sur les leviers économiques activés (Trouvé et Berriet-Solliec, 2008 ; Guérin, 2006 ; Perrin et al., 2003), soit à l'aide de modèles formels (Daniel et Kilkenny, 2009; Callois, 2006 ; Charlot, 2001). D'autres travaux empruntent aux méthodes de la micro-économétrie de l'évaluation afin de discerner ex post les effets statistiques propres d'instruments, ou même de mesures particulières d'un instrument, ayant été concrètement appliqués (Lorenceau, 2009 ; Chabé-Ferret, 2008 ; Lofredi, 2007).
} 
et politique s'attache à répondre. L'un des textes contemporains les plus importants de ce champ de réflexion est celui de John Rawls, A Theory of Justice (1971). La première section du premier chapitre s'ouvre par ces mots: "La justice est la première vertu des institutions sociales (...). Si efficaces et bien organisées que soient des institutions ou des lois, elles doivent être réformées ou abolies si elles sont injustes » (Rawls, traduction française : 1997, p. 29). Ainsi, la justice constituerait le référentiel premier pour évaluer la pertinence des objectifs d'une politique publique.

Selon Rawls, la société peut se définir comme une tentative de coopération en vue de l'avantage mutuel. Elle se caractérise à la fois par une identité d'intérêts et par un conflit d'intérêts : "Il y a identité d'intérêts puisque la coopération sociale procure à tous une vie meilleure que celle que chacun aurait eue en cherchant à viure seulement grâce à ses propres efforts. Il y a conflit d'intérêts puisque les bommes ne sont pas indifférents à la façon dont sont répartis les fruits de leur collaboration» (ib., p. 30). C'est pour résoudre ce conflit d'intérêts fondamental que des principes de justice sont nécessaires: "On a donc besoin d'un ensemble de principes pour choisir entre les différentes organisations sociales qui déterminent cette répartition des avantages et pour conclure un accord sur une distribution correcte des parts. Ces principes sont ceux de la justice sociale: ils fournissent un moyen de fixer les droits et les devoirs dans les institutions de base de la société et ils définissent la répartition adéquate des bénéfices et des charges de la coopération sociale» (ib., p. 30). D'autres problèmes sont cruciaux pour une société, tels que les problèmes de coordination, d'efficacité et de stabilité, mais le problème de la justice est prioritaire et conditionne l'atteinte de ces autres objectifs sociaux. En outre, toujours selon Rawls, une société est bien ordonnée «lorsqu'elle n'est pas seulement conçue pour favoriser le bien de ses membres, mais lorsqu'elle est aussi déterminée par une conception publique de la justice. C'est-à-dire qu'il s'agit d'une société où, premièrement, chacun accepte et sait que les autres acceptent les mêmes principes de la justice et où, deuxièmement, les institutions de base de la société satisfont, en général, et sont reconnues comme satisfaisant ces principes» (ib., p. 31). Rawls comprend dans les institutions de base de la société la constitution politique de l'Etat et l'ensemble des politiques qu'il met en œuvre. En définitive, «il est permis d'envisager cette conception publique de la justice comme constituant la charte fondamentale d'une société bien ordonnée » (ib., p. 31). Selon cette perspective, la politique rurale devrait contribuer à la mise en ouvre de la conception publique de la justice. Ses objectifs sont pertinents s'ils dérivent des principes formant la conception publique de la justice.

De fait, la société française n'est pas une société bien ordonnée; la justice fait l'objet de débats en son sein et l'Etat ne fonde pas son action sur des principes de justice explicites. De plus, Rawls propose une conception de la justice et défend l'idée que la raison devrait conduire les membres d'une société bien ordonnée à l'adopter, mais d'autres philosophes ont argumenté en faveur de conceptions différentes, qui s'y opposent plus ou moins frontalement. Dès lors, comment évaluer la pertinence des objectifs de la politique rurale sous cet angle? Dans ce qui suit, notre démarche consiste à tenter d'identifier une conception de la justice sous-jacente aux objectifs actuels de la politique rurale. Nous évaluons alors leur pertinence au regard du débat philosophique contemporain sur la justice. De manière symétrique, une seconde démarche est entreprise : considérant quelques conceptions de la justice, nous essayons d'en décliner des orientations pour la politique rurale, puis la pertinence de ces conceptions est à son tour discutée au regard du débat philosophique. Ce renversement 
de perspective correspond aussi à un renversement de problématique : la mobilisation de la philosophie morale et politique nous fait voir que le problème fondamental n'est pas tant le choix d'un référentiel permettant de juger de la pertinence des objectifs de la politique rurale, que l'absence d'une conception publique de la justice. La société bien ordonnée décrit un idéal, dont il nous semble que nous devrions essayer de nous rapprocher. Une première étape de ce rapprochement serait franchie si l'Etat explicitait la conception de la justice sur laquelle il entend fonder son action. Nous pensons que ce débat sur la justice mérite d'être posé dans le cadre du fonctionnement des institutions démocratiques, afin de mieux raisonner et justifier l'action publique. Ces éléments feront l'objet de la quatrième et de la cinquième parties de l'article. La partie suivante présente les conceptions de la justice qui serviront de base à cette discussion.

\section{Quelques conceptions philosophiques de la justice}

Nous présentons ici les grands traits d'une sélection de conceptions de la justice ${ }^{12}$. Celles-ci se rattachent schématiquement à quatre doctrines philosophiques: l'intuitionnisme, le perfectionnisme, l'utilitarisme et l'égalitarisme libéral. Avant d'entrer dans le détail, donnons en quelques mots une première idée des orientations générales que ces doctrines dessinent pour l'action publique. La doctrine intuitionniste suppose que des contradictions irréductibles se manifestent entre une pluralité de principes éthiques premiers ayant tous vocation à orienter l'action publique. Il s'agit alors pour l'Etat de trouver le juste équilibre entre ces principes. Les doctrines perfectionniste et utilitariste défendent, au contraire, l'idée qu'un principe éthique supérieur doit aider à dépasser les contractions apparentes entre principes secondaires : celui de la maximisation du bien. Ces deux doctrines ont en commun une nature téléologique - elles définissent le bien indépendamment du juste et, ensuite, définissent le juste comme ce qui maximise le bien - mais elles s'opposent fortement quant à leur définition du bien. La doctrine de l'égalitarisme libéral a, en revanche, une nature déontologique ${ }^{13}$ : le juste est défini indépendamment du bien

\footnotetext{
12 Le champ de la philosophie morale et politique est bien trop étendu pour être présenté dans son ensemble. Nous retenons ici quelques conceptions «traditionnelles» de la justice, selon le vocabulaire de Rawls, que ce dernier inclut dans les possibilités que les partenaires ayant à formuler le contrat social doivent discuter (Rawls, 1997, p. 157). Nous exposons également quelques conceptions de la justice constituant des critiques constructives de la théorie de Rawls. En revanche, nous ne présentons pas des critiques plus radicales d'inspiration libertarienne (e.g. Nozick, 1974) ou communautariste (e.g. Mac Intyre, 1981 ; Walzer, 1983). Nous nous sommes restreints également à des conceptions de nature anthropocentrique. Les conceptions biocentriques de la justice donnent, au contraire, une place centrale aux autres formes de vie, tandis que les conceptions écocentriques insistent sur le respect des équilibres écologiques (Larrère, 2006).

13 C'est-à-dire non téléologique, selon Rawls, duquel provient également la définition de la doctrine téléologique donnée immédiatement avant. Ce dernier récuse l'idée commune que les théories déontologiques sont définies comme des doctrines qui caractériseraient ce qui est juste dans les institutions et les actes indépendamment de leurs conséquences, car «toute doctrine éthique digne de considération tient compte des conséquences dans son évaluation de ce qui est juste » (Rawls, 1997, p. 55). Notons que les théories intuitionnistes peuvent être téléologiques ou déontologiques, mais sont le plus souvent déontologiques.
} 
ou ne correspond pas à la maximisation du bien. Un principe unique ou un ensemble organisé de principes guident l'Etat, qui doit œuvrer à la correction des inégalités injustes ${ }^{14}$.

\subsection{L'intuitionnisme : la politique à la recherche du juste compromis}

Dans une perspective intuitionniste, la justice consiste en la réalisation équilibrée d'un ensemble de principes éthiques premiers. Ce juste équilibre entre principes potentiellement concurrents est défini à l'aide de l'intuition et, en l'absence de consensus, à l'aide des mécanismes de décision collective. Du fait de la complexité des faits moraux, aucun principe éthique supérieur ne peut réduire ou ordonner cette pluralité. La réflexion philosophique a pour rôle d'identifier les principes premiers, mais admet «qu'il n'existe pas de réponse constructive au problème de la pondération à [leur] donner» (Rawls, 1997, p. 66). Konow (2003) propose une théorie intuitionniste mettant en balance un principe d'efficacité, un principe de besoin et un principe d'équité. Le premier requiert que la société produise la plus grande somme possible d'avantages. Le second demande que les besoins de tous les membres de la société soient satisfaits au-delà d'un niveau minimum. Le dernier impose que les avantages produits par la société soient distribués proportionnellement aux mérites de chacun. Ces trois principes ne peuvent être satisfaits simultanément et la justice consiste en un compromis. Konow montre à l'aide d'un grand nombre d'enquêtes et d'expérimentations contrôlées que cette théorie "positive » rend compte de manière large et systématique des jugements sur la justice rendus par les personnes interrogées. Rawls distingue cet «intuitionnisme de sens commun» des conceptions philosophiques intuitionnistes, qui argumentent le choix de leurs principes premiers. Une théorie substantielle met en balance deux principes premiers: «la structure de base de la société a pour but tout d'abord de produire le plus grand bien possible, au sens du plus grand solde net de satisfaction et, ensuite, de répartir de manière égale les satisfactions» (Rawls, 1997, p. 62). Cette dichotomie entre «masse » et «répartition» inspire directement l'économie publique, lorsque cette dernière présente l'arbitrage entre efficacité et équité comme constituant le dilemme fondamental de la décision publique.

\subsection{Perfectionnisme et utilitarisme : la politique pour promouvoir le bien}

\subsubsection{Le perfectionnisme}

Dans une perspective perfectionniste, le bien consiste en ce qu'il y a d'excellent dans l'homme. La conception du bien est objective : ce qui est considéré comme excellence vaut pour tous et indépendamment de l'opinion de chacun. Des théories perfectionnistes mettront en exergue, par exemple, l'expression de certaines vertus, les

\footnotetext{
14 Cette classification est schématique, car des théories relevant de l'égalitarisme libéral, de même que des théories perfectionnistes pourraient être intuitionnistes. Rawls présente ce dernier cas (ib., p. 66) : la maximisation du bien est requise, mais les règles permettant d'estimer ce qui produit le plus de bien ne sont pas définies et il est nécessaire de faire usage de l'intuition pour mettre en balance plusieurs valeurs perfectionnistes contribuant au bien (la création de la beauté, la recherche du savoir, etc.).
} 
grandes réalisations artistiques, scientifiques ou religieuses des hommes, etc. Une fois ces excellences définies, les interventions publiques nécessaires à leur sauvegarde ou à leur développement devront être prioritaires sur toutes celles qui concernent la satisfaction des "plaisirs inférieurs", si répandus que soient ces derniers. Le perfectionnisme érigé en principe politique est une extension de l'éthique des vertus aristotélicienne, qui prône la recherche individuelle de la perfection morale, et de la pensée de Nietzsche, qui exalte la morale des forts en opposition à celle des faibles.

\subsubsection{L'utilitarisme}

Dans une perspective utilitariste, le bien consiste en l'utilité. Deux interprétations principales sont données à ce concept : il s'agit du niveau de plaisir éprouvé par une personne dans sa vie, net des peines qu'elle a endurées, ou alternativement du niveau de satisfaction d'une personne quant à la réussite de sa vie, évalué globalement ou au regard du degré de réalisation de son projet de vie. Dans tous les cas, la conception utilitariste du bien est subjective en ce sens que la quantité de bien dont dispose une personne ne peut être évaluée que par elle-même. Les interventions publiques doivent favoriser la satisfaction des désirs des membres de la société, en ne tenant compte que de l'utilité et sans émettre de jugements perfectionnistes sur leurs qualités intrinsèques. C'est en cela que l'utilitarisme est libéral. Cette doctrine remonte aux travaux de Bentham. Ce dernier pense que la recherche d'utilité est l'élément fondamental de la vie de chacun, toutes les autres valeurs n'étant qu'instrumentales. Puisque ce principe est valable à l'échelle de l'homme, il doit l'être aussi à l'échelle de la société dans son ensemble, qu'il considère en tant que corps social. Le législateur doit rechercher «le plus grand bonheur pour le plus grand nombre ». Il devrait agir à la manière d'un spectateur impartial de la société, qui «sympathiserait " ${ }^{15}$ également avec tous les plaisirs et les peines de chacun de ses membres et chercherait de ce fait à organiser les institutions de manière à ressentir la plus grande somme nette de plaisirs. Dans le calcul de l'utilité sociale, le même poids est donné à l'utilité de chacun des membres de la société. C'est cette impartialité qui fonde le caractère équitable de l'objectif utilitariste.

\subsection{L'égalitarisme libéral : la politique contre les inégalités injustes}

\subsubsection{La théorie de Rawls}

Dans une perspective rawlsienne, la justice consiste en une répartition équitable des «biens sociaux premiers» entre les citoyens et les groupes sociaux. Les biens sociaux premiers sont les libertés, possibilités, droits, revenus, richesses et base sociale du respect de soi ${ }^{16}$. Les groupes sociaux sont définis en référence aux

\footnotetext{
15 Selon cette acception du terme «sympathie » : participation à la joie ou à la peine d'autrui (Larousse.fr).

16 Ces ressources sont "premières ", car utiles à chacun pour définir et réaliser son projet de vie propre. Elles sont «sociales », car les institutions de base de la société (constitution et législation) sont en mesure d'en organiser la répartition. Rawls a ajouté le loisir dans un deuxième temps.
} 
positions sociales, c'est-à-dire aux positions de la structure sociale qui offrent aux hommes qui y naissent des perspectives de vie différentes. Deux principes d'égalisation sont proposés. Le premier est prioritaire sur le second et concerne tous les citoyens de manière égale. Il requiert la stricte égalisation des libertés formelles ${ }^{17}$. Le second traite de la répartition entre groupes sociaux de tous les autres biens sociaux premiers ${ }^{18}$. Il se compose lui-même de deux principes ordonnés. Le premier est le principe d'égalisation des chances d'accès aux fonctions et aux positions sociales, d'après lequel les différences de dons naturels et de motivation devraient être les seules à intervenir à ce niveau, indépendamment du point de départ de chacun dans la société. Le second est le principe de différence, selon lequel les inégalités équitables entre groupes sociaux sont celles qui améliorent les perspectives à long terme d'accès aux biens sociaux premiers des membres du groupe social le plus désavantagé ${ }^{19}$. L'égalitarisme rawlsien est libéral, car il reconnait explicitement la pluralité des personnes et de leurs conceptions du bien: en vertu du premier principe et à l'aide des ressources attachées à son point de départ dans la structure sociale, chaque personne est libre de définir et de poursuivre son projet de vie propre. A cette liberté est associée une responsabilité : la focalisation sur les ressources rend chaque personne responsable de ses ambitions et, donc, de l'utilité qu'elle se procure au final. Cette théorie s'inscrit dans la filiation de la théorie traditionnelle du contrat social de Locke, Rousseau et Kant.

\subsubsection{Les théories de l'égalité des chances}

Dans une perspective d'égalité des chances ${ }^{20}$, la justice se focalise sur les opportunités qui s'offrent aux individus et donne ainsi une place centrale à leur capacité à faire des choix. Elle requiert le «laisser faire » vis-à-vis des inégalités qui sont le produit des choix passés et la compensation des inégalités résultant de circonstances non choisies. Plusieurs théories se distinguent par leur définition du choix et de la nature de ce qu'elle demande d'égaliser. Pour Dworkin (1981, 2000), le choix correspond à l'expression des préférences individuelles et il s'agit d'égaliser l'ensemble des ressources externes (positions sociales, richesses, etc.) et internes (talents, handicaps) dont disposent les individus du fait de circonstances non choisies. Pour Arneson (1989),

\footnotetext{
17 Il s'agit des libertés de jure, garanties formellement par le droit, idéalement par la constitution. 18 Y compris des possibilités réelles qu'offrent les libertés formelles.

19 Formellement, le principe de différence contient un juste principe d'épargne qui garantit la prise en compte des générations futures : «chaque génération doit, non seulement, conserver les acquisitions de la culture et de la civilisation et maintenir intactes les institutions justes qui ont été établies, mais elle doit aussi mettre de côté, à chaque période, une quantité suffisante de capital réel accumulé. Cette épargne peut prendre des formes diverses, depuis l'investissement net dans les machines et les autres moyens de production jusqu'aux investissements en culture et en éducation » (Rawls, 1997, p. 325). En suivant cette logique, il faut sans conteste ajouter à ce niveau la préservation du capital naturel.

20 Cette dénomination est utilisée pour simplifier l'exposé, mais dans la littérature philosophique, la théorie de Dworkin est qualifiée de «théorie de l'égalité des ressources ", celles d'Arneson et Cohen de «théories de l'égalité des opportunités» et celle de Sen de «théorie de l'égalité des capabilités ».
} 
Cohen (1989) et Sen (1985) ${ }^{21}$, seuls les choix véritables doivent entrer en ligne de compte, c'est-à-dire les décisions (contrefactuelles) qui auraient été prises en l'absence d'influences sociales et d'autres contraintes non choisies. Pour Arneson, l'idéal distributif est l'égalisation des opportunités d'utilité totale: à l'entrée dans leur vie d'adulte, les individus devraient être en mesure d'effectuer des choix véritables au sein d'ensembles d'opportunités équivalents, c'est-à-dire dont les options de même rang, lorsqu'elles sont ordonnées du point de vue de l'utilité totale qu'elles procurent, ont des valeurs égales. Sen base sa théorie sur les concepts de «fonctionnement» et de «capabilité ». Les fonctionnements sont des éléments objectifs qui se situent entre les ressources et l'utilité, « ce que les biens peuvent faire pour les gens », c'est-à-dire toutes les possibilités d'être et de faire : être en bonne santé, bien logé, travailler, voyager, etc. La capabilité d'une personne est l'ensemble des vecteurs de fonctionnements qui lui sont accessibles, au sein duquel elle peut choisir la vie qu'elle souhaite mener. La justice consiste en l'égalisation des capabilités ${ }^{22}$, autrement dit de la liberté effective de choisir sa vie. Ces conceptions sont issues d'une critique de la place de la liberté (pour Sen) ou de la responsabilité (pour les autres) dans la théorie de Rawls.

Ces conceptions de la justice servent de base aux développements de la partie suivante. Nous avons mis volontairement de côté ce qui constitue véritablement le cœur de la philosophie morale et politique - le débat critique entre les défenseurs de ces conceptions - auquel nous revenons dans la dernière partie.

\section{Les fondements de la politique rurale}

Cette quatrième partie est consacrée aux rapports que l'on peut établir entre les orientations de la politique rurale et les principes de la justice sociale. Dans une première section, nous nous demandons si les orientations de la politique actuellement en vigueur se justifient au regard d'une conception de la justice philosophiquement établie. Disons d'emblée que s'il s'agit d'identifier un soubassement théorique précis sur lequel s'adosseraient les objectifs actuels de la politique rurale, la réponse est clairement négative. Toutefois, nous défendons l'idée que ces derniers forment un ensemble qui s'apparente à une conception ad hoc de la justice de nature intuitionniste. Dans les sections qui suivent, nous posons la question symétrique de savoir, considérant une conception de la justice donnée, s'il est possible d'établir des orientations concordantes de la politique rurale. En supposant que les pouvoirs publics adoptent une conception de la justice, la démarche d'élaboration de la politique rurale consisterait à spécifier ses objectifs et à définir ses modalités d'action sur la base d'une analyse de leur contribution à la réalisation des principes de la justice. Cette démarche est esquissée successivement pour plusieurs conceptions de la justice présentées dans la partie précédente. Notre propos a valeur d'illustration, puisqu'il s'agit d'une sélection

\footnotetext{
21 Voir également Roemer (1998).

22 Cohen propose un principe similaire d'égalisation de l'accès aux avantages. Le changement de vocabulaire vise à souligner, d'une part, l'importance d'égaliser aussi les capacités des individus à saisir les opportunités disponibles (l'accès) et, d'autre part, l'inclusion de l'utilité parmi les fonctionnements (les avantages).
} 
de conceptions de la justice et d'hypothèses d'école discutables sur les modalités de la politique rurale susceptibles de contribuer à leur réalisation. Il permet néanmoins de "vérifier » que l'effort d'explicitation de la conception de la justice sous-jacente à l'action publique porterait à conséquence du point de vue des orientations générales de la politique rurale.

\subsection{La politique rurale actuelle : des fondements de nature intuitionniste}

L'examen du plan stratégique national et de l'exposé des motifs de la loi relative au développement des territoires ruraux a permis d'identifier les objectifs assignés par les pouvoirs publics à la politique rurale. A l'échelle des territoires ruraux, la finalité première est la valorisation économique, sociale et environnementale. A l'échelle nationale, les objectifs poursuivis sont la croissance économique et la création d'emploi, la cohésion sociale et territoriale, la justice sociale et l'équité territoriale, la préservation de la diversité des territoires et la durabilité environnementale. Il apparaît clairement que cet ensemble d'objectifs ne se fonde pas sur une conception de la justice philosophiquement établie. Il semble plutôt correspondre au résultat d'additions successives d'objectifs, partant d'objectifs historiques, sous l'influence de courants de pensée scientifiques ou politiques (théories du développement local, nouvelle économie géographique, développement durable, etc.), de l'évolution du contexte institutionnel national et européen, ainsi que de l'évolution des objectifs des politiques nationales et communautaires en relation avec le développement rural.

Cela étant, les caractéristiques de cet ensemble d'objectifs s'apparentent à celles d'une conception intuitionniste de la justice. Les objectifs sont multiples, potentiellement antagonistes et essentiellement juxtaposés les uns aux autres. La recherche de leur réalisation simultanée produit nécessairement des tensions et demande des arbitrages. Si ces arbitrages étaient inadéquats (si, par exemple, la durabilité environnementale était systématiquement sacrifiée au profit de la maximisation de la croissance économique à court terme), ces orientations perdraient toute leur pertinence. Elles n'ont donc de valeur qu'à une condition : qu'un compromis approprié entre objectifs, tenant compte simultanément des échelles locale et nationale, soit formulé. Or, l'Etat ne propose pas de règles pour définir les termes de ce compromis : il s'en remet à l'intuition partagée ou aux rapports de force entre acteurs impliqués dans sa négociation et se contente d'identifier ces derniers: «les collectivités publiques apparaissent souvent comme les mieux placées pour gérer les spécificités, l'Etat doit veiller à redonner toute sa place à l'expertise locale (...). Cependant, l'Etat doit veiller à assurer la cohérence d'ensemble des politiques » (Exposé des motifs de la loi relative au développement des territoires ruraux, 2005); "L'Union européenne est désormais un intervenant important de la politique régionale et de la politique en faveur des zones rurales. Au plan national, la région et le département deviennent les lieux privilégiés de définition des besoins et de programmation des politiques » (MAP, 2007). On reconnaît les caractéristiques de la doctrine intuitionniste présentée précédemment.

Les éléments historiques rappelés dans la partie 2 montrent que cette dimension intuitionniste de la politique rurale n'est pas nouvelle. Il faut souligner cependant la complexité croissante associée à la formulation d'un compromis entre objectifs. Celle-ci 
est due, en premier lieu, à l'élargissement du nombre d'objectifs mis en balance: les orientations de la politique rurale se raisonnaient après-guerre essentiellement sur la base de la dichotomie classique entre masse et répartition (maximisation de la croissance économique $v$ s égalisation des richesses), alors qu'elle implique aujourd'hui l'ensemble des buts sociaux identifiés ci-dessus. Elle est due également à l'augmentation du nombre de participants à sa négociation: du quasi-monopole de l'Etat central après-guerre à la concertation des multiples échelons d'administration territoriale et des acteurs des territoires de nos jours. Enfin, bien que l'Etat fasse l'effort d'énoncer des objectifs généraux pour la politique rurale, l'arbitrage entre ces derniers n'est jamais discuté qu'au niveau des instruments de sa mise en œuvre. L'augmentation continue de leur nombre durant la période considérée rend d'autant plus délicate l'obtention d'une vision d'ensemble sur le compromis obtenu.

Le FEADER illustre, de manière relativement transparente, ce processus d'arbitrage entre les objectifs de la politique rurale à l'échelle d'un programme particulier. Dans le plan stratégique national (PSN), l'Etat assigne à ce programme les mêmes objectifs qu'à la politique nationale globale de développement rural, c'est-àdire, au premier chef, "valoriser les territoires ruraux du point de vue économique, social et environnemental $» 23$. A chacune de ses trois dimensions de création de valeur est consacré - même si la correspondance n'est pas stricte - un axe du programme, regroupant un certain nombre de mesures concrètes. L'objectif opérationnel du premier axe est «l'amélioration de la compétitivité des secteurs agricoles et forestiers »; celui du second «l'amélioration de l'environnement de la gestion de l'espace »; et celui du troisième "l'amélioration de la qualité de vie en milieu rural et la diversification de l'économie rurale » 24 . Ces trois axes sont prédéfinis dans les orientations stratégiques communautaires de l'Union européenne (Conseil de l'Union européenne, 2006), que le PSN doit respecter. La construction d'un compromis entre les objectifs de valorisation économique, sociale et environnementale est en partie effectuée à travers la négociation des volumes financiers attribués à chaque axe. Une première négociation au niveau communautaire a conduit à l'affectation à chaque axe de pourcentages minimum, respectivement $10 \%, 25 \%$ et $10 \%$. Ensuite, le débat national a permis d'arrêter des cibles plus précises, à savoir $39 \%, 49 \%$ et $11 \%$. A un niveau plus fin, des cibles ont été arrêtées également pour chacune des trois ou quatre priorités établies au sein des axes. La négociation régionale est finalement responsable des affectations entre mesures au sein de ces priorités. Cette procédure d'arbitrage explicite n'est toutefois pas représentative des démarches habituelles. Dans le cas de la loi relative au développement des territoires ruraux, nous avons vu que l'organisation des dispositions législatives présente également une certaine correspondance avec la structure des objectifs visés ${ }^{25}$. Cependant, aucune indication n'est donnée sur l'importance relative de ces différentes dispositions, donc sur le poids attribué à chacun de ces objectifs visés.

\footnotetext{
23 Cf. section 2.2 .

${ }^{24} \mathrm{~A}$ ces trois axes s'ajoute un quatrième, transversal, consacré à l'approche LEADER, c'est-à-dire "une méthode de mise en œuvre qui permet d'accompagner les stratégies de développement de partenariats locaux » (MAP, 2007, p. 30).

${ }_{25} \mathrm{Cf}$. section 2.2 .
} 
Pour conclure cette section, il nous faut souligner que l'ensemble formé par les objectifs de la politique rurale ne fait que «s'apparenter» à une conception intuitionniste de la justice. D'une part, le choix des objectifs n'est pas justifié par une argumentation philosophique et, le plus souvent, ces derniers ne sont pas clairement définis. Que signifient en effet précisément les notions de "cohésion sociale " et «d'équité territoriale », et pourquoi faudrait-il poursuivre ces objectifs? D'autre part, «la justice sociale » est évoquée comme un objectif parmi d'autres et il n'est précisé nulle part que la politique rurale doit contribuer dans son ensemble à la réalisation de la justice. La suite de notre discussion sera contrefactuelle. Que se passerait-il si les objectifs de la politique rurale étaient véritablement définis en référence à une conception philosophique de la justice? Quelles orientations de la politique rurale pourraient être dérivées, en particulier, de la mise en œuvre d'une théorie de la justice perfectionniste, utilitariste ou égalitariste?

\subsection{Esquisse d'une politique rurale perfectionniste}

Supposons que l'Etat adopte une conception perfectionniste de la justice. Plus précisément, supposons qu'il admette que sa finalité supérieure est de contribuer à la préservation et à l'expansion du patrimoine culturel national. Nous pouvons imaginer comment celui-ci s'organiserait en conséquence. Par exemple, une commission nationale composée de parlementaires, de bureaucrates ou d'experts indépendants aurait pour mission permanente d'identifier les éléments ayant vocation à être pris en compte dans l'évaluation de ce patrimoine. Suivant ses avis, les ministères seraient en charge de la conception des politiques visant à assurer à long terme leur préservation ou leur promotion. Alternativement, un mode d'organisation décentralisé pourrait être institué, chaque région ou territoire étant en charge de la définition et de la mise en œuvre de ses politiques, sous l'hypothèse que cette décentralisation conduirait à une meilleure préservation et à un plus grand développement de ce patrimoine.

Dans ce contexte, la politique rurale devrait admettre pour finalité première d'assurer une contribution optimale des territoires ruraux à la préservation et au développement du patrimoine culturel national. Sa principale modalité d'action devrait être, très certainement, le soutien à la préservation des singularités culturelles des territoires ruraux. Du fait de leur relatif isolement des grands centres de population et leur environnement immédiat marqué par un faible degré d'artificialisation, les communautés rurales sont susceptibles de faire vivre des pratiques économiques et sociales originales. Certaines d'entre elles pourraient être considérées comme des productions culturelles de valeur et être soutenues à ce titre. Les paysages ruraux, résultant de l'association de modes d'habitat et d'éléments architecturaux typiques avec des pratiques agricoles spécifiques, en sont une illustration emblématique. Des langues vernaculaires, des pratiques folkloriques ou artisanales locales en constituent d'autres exemples. Les spécificités associées aux modes de vie quotidiens des ruraux pourraient également en faire partie. A côté de cette première modalité, portant directement sur la «fonction culturelle» des territoires ruraux, d'autres modalités d'action plus indirectes porteraient sur leurs fonctions économique, sociale et écologique, qui conditionnent à long terme le développement culturel des communautés rurales et de la société nationale. Le soutien aux projets de valorisation des potentialités 
économiques, sociales et environnementales des territoires ruraux, par exemple, constituerait vraisemblablement une modalité d'action de cette politique, strictement encadrée à l'aune de son impact de long terme sur le patrimoine culturel. Le rééquilibrage d'ensemble de la géographie nationale des activités et des services, en revanche, serait probablement rejeté, car il risquerait d'aller dans le sens de l'uniformisation des modes de consommation et de travail sur le territoire national, contribuant ainsi à la disparition des spécificités culturelles rurales.

\subsection{Esquisse d'une politique rurale utilitariste}

Supposons que l'Etat adopte une conception utilitariste de la justice. La finalité des politiques publiques serait, par exemple, de contribuer à la maximisation de la somme totale d'utilité dont bénéficie l'ensemble des membres des générations actuelles et futures. Nous pouvons envisager deux traductions opératoires de cette finalité sociale: (i) une option simplificatrice serait de retenir que l'utilité est un niveau de satisfaction tiré du revenu et que l'utilité marginale apportée par une unité de revenu supplémentaire est identique pour tous les individus, quel que soit leur niveau de revenu, leurs autres caractéristiques individuelles et quel que soit le moment au cours de leur vie où ils la recevront; l'objectif social correspondrait alors à la maximisation du revenu total produit par l'économie nationale sur la période considérée, et nous pouvons admettre que la prise en compte de l'avenir s'arrêtera dans les faits aux quelques générations immédiatement à venir ; (ii) une option plus fidèle à la théorie consisterait à tenter d'établir un indicateur statistique représentatif du niveau de satisfaction sociale, afin d'évaluer au mieux l'impact à long terme des politiques sur ce dernier.

Si la première option était retenue, la politique rurale devrait admettre pour finalité première d'assurer une contribution optimale des territoires ruraux à la maximisation à long terme du revenu national. Ses principales modalités d'action seraient sans doute le soutien aux projets de valorisation à long terme des potentialités économiques des territoires ruraux, ainsi que le soutien aux fonctions rurales d'appui à la croissance urbaine de long terme. Les territoires ruraux assurent historiquement une fonction productive, en particulier par la valorisation agricole et sylvicole de leurs ressources fixes naturelles, mais aussi grâce à la présence d'industries s'appuyant sur des savoir-faire locaux particuliers. Ces secteurs d'activités sont certainement en mesure de contribuer au développement économique national, bien que leur capacité à valoriser les ressources naturelles et culturelles de manière durable demanderait à être améliorée. Des opportunités de croissance durable du revenu pourraient également être recherchées sur la base des fonctions résidentielles et récréatives de l'espace rural, et ceci d'autant plus que les aménités naturelles prennent une importance grandissante pour les ménages. Dans tous les cas cependant, il faudrait être attentif au fait que la croissance économique d'un territoire ne soit pas obtenue au détriment de la croissance d'autres territoires, conduisant ainsi à un effet nul, voire négatif, au niveau national. Parallèlement, les territoires ruraux sont les plus à même d'assurer certaines fonctions écologiques essentielles à la durabilité de la croissance urbaine : régulation des masses d'eau afin d'éviter des inondations destructrices, stockage du carbone afin de limiter le réchauffement climatique, etc. Ils sont également en capacité d'atténuer certains 
facteurs limitant du développement urbain, en particulier la contrainte de la congestion foncière, en assurant une fonction d'accueil de grandes infrastructures, tels que, par exemple, les centrales de production d'énergie, les centres d'élimination des déchets, les plateformes logistiques, etc.

Qu'en serait-il si la seconde option était retenue? La politique rurale devrait admettre pour finalité première d'assurer une contribution optimale des territoires ruraux à la maximisation à long terme de l'indice d'utilité sociale. Cet objectif differe du précédent, car il paraît peu probable que l'augmentation du revenu total conduise à l'augmentation de la satisfaction totale. Les hypothèses sur l'utilité et l'utilité marginale formulées dans le cas de l'option simplificatrice ne sont vraisemblablement pas vérifiées dans les faits ${ }^{26}$. Tout d'abord, les individus tirent leur satisfaction de la consommation de nombreux biens non marchands - l'usage du terme de ressource permettra de garder ce point à l'esprit. Ensuite, l'utilité marginale est sans aucun doute décroissante avec le niveau de ressources: il y a saturation progressive des besoins. Enfin, les individus n'ont pas les mêmes capacités de conversion des ressources disponibles en satisfaction: des facteurs individuels (état d'esprit...), géographiques (accessibilité...) et sociaux (normes sociales...) interviennent à ce niveau. Ainsi, si l'indice d'utilité sociale dépend toujours de la somme totale de revenus, il dépend aussi de la répartition interindividuelle des ressources et des capacités de conversion. Il est donc lié aux caractéristiques territoriales intervenant à ce niveau. De ce point de vue, malgré l'avantage de moindres prix fonciers, les territoires ruraux paraissent dans l'ensemble dotés de caractéristiques défavorables pour leurs habitants. L'éloignement des agglomérations les pénalise, en termes d'accès aux lieux centraux de formation, d'emploi, de consommation, de culture, d'expression politique, ainsi qu'aux services supérieurs de santé. Ils se trouvent désavantagés également en termes de salaire, toutes choses égales sur le plan de leurs caractéristiques individuelles. S'ajoute à ces caractéristiques territoriales défavorables, une inégalité territoriale en termes de composition sociale, la population rurale étant dans l'ensemble moins «favorisée » que la population urbaine sur ce plan. Au final, il est probable que la population rurale dispose en moyenne d'un niveau de ressources et de satisfaction plus faible que la population urbaine et, en définitive, d'un niveau d'utilité marginale plus élevé : des transferts de ressources de la population urbaine vers la population rurale ou une action de rééquilibrage d'ensemble de la géographie nationale des services et des activités pourraient alors conduire à une augmentation à long terme de l'indice d'utilité sociale ${ }^{27}$. Cette redistribution territoriale constituerait alors une modalité d'action importante de la politique rurale, bien qu'elle risquerait de s'opposer, dans une certaine mesure, à l'amélioration générale du revenu.

\footnotetext{
${ }_{26}$ Nous évacuons dans ce qui suit la question de la prise en compte du temps, d'une grande complexité.

27 Ces transferts contribueraient à court terme à l'égalisation des utilités marginales individuelles, donc à la maximisation de l'utilité totale à court terme, mais leurs effets indirects sur le système d'incitation économique et la production totale de ressources devraient également être envisagés pour garantir la prise en compte du long terme.
} 


\subsection{Esquisse d'une politique rurale rawlsienne}

Supposons maintenant que l'Etat adopte une conception égalitariste de la justice ou, plus exactement, qu'il admette que la finalité de la politique rurale est de contribuer à la réalisation du second principe de justice de Rawls ${ }^{28}$. Supposons également que le débat national ait permis de s'entendre sur la définition du groupe social le plus défavorisé et sur la construction de l'indice des biens sociaux premiers: par exemple, le premier regroupe tous ceux qui ont moins de la moitié du revenu médian ${ }^{29}$ et le second ne tient compte que du niveau de richesse des individus, sous l'hypothèse que les autres biens sociaux premiers y sont suffisamment corrélés.

Selon cette perspective, la politique rurale devrait admettre pour finalité première d'assurer une contribution optimale des territoires ruraux à l'égalisation des chances d'accès aux positions sociales entre groupes sociaux et à la maximisation des perspectives à long terme des membres du groupe social le plus désavantagé dans la communauté nationale. En premier lieu, la recherche de l'égalité des chances d'accès aux positions sociales imposerait certainement une action de rééquilibrage de la géographie nationale des activités et des services ou, plus généralement, de redistribution des ressources en faveur des habitants des territoires ruraux. Elle demanderait, en parallèle, une action en faveur de la réduction des contraintes pesant sur les mobilités résidentielles entre territoires. En effet, les désavantages associés aux localisations rurales, en termes de salaires et d'accès aux lieux centraux de formation, d'emploi, de consommation, de culture, d'expression politique et de santé, se traduisent vraisemblablement sur le plan de l'inégalité des chances. Ils affectent négativement l'acquisition de capital humain et social des enfants qui grandissent dans ces territoires, réduisant ainsi leurs chances d'accéder un jour aux fonctions et positions sociales qui requièrent intensivement ces capitaux. De plus, associés à l'ensemble des contraintes psychologiques, informationnelles et financières qui font barrière aux mobilités résidentielles, ils réduisent les chances des adultes qui habitent ces territoires, toutes choses égales du point de vue de leurs motivations et de leurs efforts, d'accéder aux nombreuses fonctions et positions sociales localisées dans les territoires urbains. Etant donné les différences de composition sociale entre territoires ruraux et urbains, ces inégalités des chances entre "groupes territoriaux», définis sur la base de la localisation de leurs membres, vont se traduire dans le long terme sur le plan de l'inégalité des chances entre "groupes sociaux », définis au niveau national d'après leurs richesses respectives. On peut s'attendre à ce qu'elles contribuent dans l'ensemble à aggraver ce problème, justifiant ainsi les modalités d'action mentionnées. En second lieu, la mise en œuvre du principe de différence devrait probablement s'appuyer sur des modalités très diverses mettant à contribution l'ensemble des fonctions de l'espace rural. Leurs effets devraient être suivis de près, en gardant à l'esprit qu'il ne s'agit en soi ni d'augmenter les ressources totales, ni de réduire l'inégalité sociale ou territoriale,

\footnotetext{
${ }^{28}$ Le premier principe de Rawls ne peut justifier une politique rurale. Il demande que tous les citoyens bénéficient de libertés formelles égales, garanties par exemple par la constitution politique de l'Etat. Les possibilités réelles qu'offrent ces droits formels peuvent s'avérer inégales pour les individus, mais il s'agit alors d'un problème de répartition qui concerne le second principe.

29 Il s'agit d'une proposition faite par Rawls (1997, p. 128).
} 
ni même spécifiquement d'améliorer l'accès aux ressources des personnes les plus défavorisées des territoires ruraux; ces buts secondaires devraient être poursuivis et pondérés, de manière à atteindre l'objectif supérieur de la réalisation à l'échelle nationale de la justice sociale, par l'amélioration des perspectives de long terme du groupe social le plus désavantagé, en le considérant dans son ensemble.

\subsection{Esquisse d'une politique rurale d'égalisation des chances}

Supposons enfin qu'une théorie de l'égalité des chances soit adoptée par l'Etat, à savoir celle proposée par Dworkin, qui requiert l'égalisation de l'ensemble des ressources dont les individus disposent du fait de circonstances non choisies ${ }^{30}$. Suivant les conseils de cet auteur, l'Etat soutiendrait autant que possible l'extension du marché concurrentiel des assurances, afin que soit couvert le plus grand nombre possible de "sinistres", c'est-à-dire d'évènements probables ayant des conséquences négatives sur les ressources des individus. En effet, ce marché permet l'expression des préférences individuelles concernant le niveau de couverture de chaque risque, donc le niveau des primes d'assurance à payer. Il autorise ainsi un choix là où il n'y en a pas en son absence, et met tous les individus à égalité devant ce choix ${ }^{31}$. Puisque les inégalités qui résultent des choix sont équitables, les conséquences de ces sinistres ne constituent plus un problème pour la justice. Cependant, les «sinistres » qui adviennent dès la naissance ou durant l'enfance ne peuvent pas faire l'objet d'un choix d'assurance par les intéressés. Cette fois, l'Etat ferait appel à la fiscalité. Pour définir l'ampleur d'une couverture publique équitable, il lui faudrait imaginer et mimer les résultats d'un marché d'assurance hypothétique, auquel les individus auraient virtuellement accès avant même leur naissance.

Dans ce contexte, la politique rurale devrait admettre pour finalité première d'assurer une contribution optimale des territoires ruraux à la correction des inégalités résultant de circonstances non choisies. Une première modalité d'action éventuelle correspondrait à la correction des défaillances ou imperfections du marché des assurances privées spécifiques aux territoires ruraux. Une seconde modalité constituerait à assurer une redistribution territoriale en faveur des territoires ruraux, couvrant le « risque » d'une enfance rurale. En effet, nous avons déjà indiqué qu'un certain nombre de désavantages associés aux localisations rurales sont susceptibles d'affecter négativement l'acquisition de capital humain et social des enfants qui grandissent dans ces territoires. La question pertinente du point de vue de la justice est alors de savoir quel niveau de couverture serait préféré par un individu moyen, s'il avait la possibilité de se prémunir, avant même sa naissance, contre cette éventualité. Des actions de rééquilibrage d'ensemble de la géographie des activités et des services et des transferts directs de ressources en faveur des habitants des territoires ruraux devraient être définies en conséquence. Enfin, une troisième modalité d'action serait d'assurer à

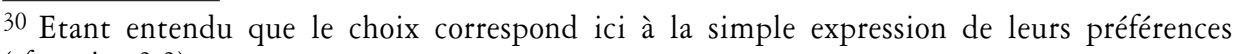
(cf. section 3.3).

31 A condition que la répartition des revenus soit équitable au moment de ce choix, donc que la justice ait été assurée en amont ( $c f$. la fin du paragraphe).
} 
chacun, à l'entrée dans la vie d'adulte et à ce moment-là uniquement, la possibilité de choisir la localisation territoriale correspondant pleinement à ses préférences. La localisation initiale d'un jeune adulte est une circonstance non choisie, et même si les désavantages associés à l'enfance rurale sont compensés, une localisation initiale rurale conduira possiblement à des désavantages ultérieurs. Pour que les inégalités associées aux localisations initiales soient équitables, il est nécessaire que chaque individu dispose du choix de son implantation initiale. Une fois ce choix effectué et réalisé, ses conséquences pour le reste de la vie adulte ne concernent plus la justice.

Dans cette partie, nous avons entrepris deux démarches. D'une part, nous avons identifié les fondements philosophiques des orientations actuelles de la politique rurale française: ses objectifs forment un ensemble qui s'apparente à une conception intuitionniste de la justice. D'autre part, nous avons imaginé des orientations fondées sur d'autres conceptions de la justice: elles apparaissent sensiblement différentes des premières. Nous pouvons maintenant revenir à la question de l'évaluation de la pertinence des objectifs de la politique rurale.

\section{Le débat sur la justice}

La pertinence des objectifs de la politique rurale dépend de la pertinence de la conception de la justice sur laquelle ils se fondent: quelle est la validité de la doctrine intuitionniste qui sous-tend les orientations actuelles de la politique rurale, et plus généralement, quelle est celle des différentes conceptions de la justice auxquelles nous avons fait appel afin d'esquisser des orientations alternatives? Ce questionnement fait l'objet de cette dernière partie. Le débat critique entre théoriciens de la justice est au cœur des travaux de philosophie morale et politique. Nous présentons ici quelques éléments marquants de ce débat ayant trait aux conceptions mobilisées précédemment, en distinguant la critique des théories traditionnelles de celles inspirées par la théorie de Rawls. L'examen de ce champ de réflexion nous conduit à penser, au-delà de la question de la pertinence d'une politique particulière, que le problème fondamental consiste en l'adoption par l'Etat d'une conception de la justice sur laquelle fonder son action. L'organisation de notre propos pouvait suggérer jusqu'à présent un certain relativisme éthique : la mobilisation successive de différentes conceptions de la justice a pu laisser penser que toutes se valent pour orienter la politique. Nous souhaitons montrer ici que l'essentiel, mais aussi le plus difficile, est d'arbitrer entre ces conceptions.

\subsection{La critique des théories traditionnelles}

\subsubsection{Critiques de l'intuitionnisme}

L'intuitionnisme reconnait une pluralité de principes premiers et fait l'hypothèse que l'intuition sur la justice est suffisamment commune aux hommes pour qu'un consensus approprié puisse émerger sur le problème de leur pondération. Le problème principal de cette doctrine tient à la fragilité de cette hypothèse. Rawls pense que l'intuition est trop susceptible d'être biaisée par l'habitude et l'intérêt personnel pour qu'une place de cette importance puisse raisonnablement lui être octroyée: le compromis entre principes 
premiers a de fortes chances de refléter l'équilibre des positions relatives de pouvoir plutôt que la justice. Bien qu'une «conception de la justice, quelle qu'elle soit, doit faire confiance à l'intuition dans une certaine mesure (...,), nous devrions faire notre possible pour limiter ce recours direct à nos jugements bien pesés »(Rawls, 1997, p. 67). Il reconnaît luimême la difficulté de l'entreprise : «nous devons admettre la possibilité de n'arriver à rien de mieux qu'une pluralité de principes» (ib., p. 67). Toutefois, il pense que régler le problème de la priorité entre buts sociaux est un défi fondamental que doit relever la philosophie politique. L'intuitionnisme pourra être rejeté si la réflexion philosophique parvient à "présenter cette sorte de critères constructifs qui, d'après lui, n'existent pas» (ib., p. 65). Ainsi, la question du crédit à accorder à la doctrine intuitionniste dépend au premier chef du crédit qu'il est possible d'accorder aux théories non intuitionnistes.

\subsubsection{Critiques du perfectionnisme et de l'utilitarisme}

Les doctrines perfectionniste et utilitariste répondent au problème posé par l'intuitionnisme en élevant un principe à un rang supérieur : celui de la maximisation du bien. Les critiques portent alors sur la définition du bien et sur le principe de maximisation. Dans le cas du perfectionnisme, le bien est défini objectivement et vaut pour tous. En faisant la promotion de ce bien, les institutions sociales favorisent les desseins de certains aux dépens d'autres : leur impartialité n'est donc pas assurée. C'est pourquoi le perfectionnisme est largement rejeté en tant que principe de justice. Dans le cas de l'utilitarisme, le bien est subjectif: le soin est laissé à chacun d'évaluer la quantité d'utilité dont il dispose. Les institutions sociales sont impartiales de ce point de vue, parce qu'elles donnent le même poids à l'utilité de chacun dans la somme totale. Néanmoins, une dimension perfectionniste subsiste, car cette théorie implique que l'utilité est le seul bien important pour les hommes. En outre, la focalisation sur la somme totale pose problème, car elle n'offre «aucune raison de principe pour laquelle les gains de certains ne compenseraient pas les pertes des autres, (nit) pour laquelle la violation de la liberté d'un petit nombre ne pourrait pas être justifiée par un plus grand bonbeur pour un plus grand nombre » (Rawls, 1997, p. 52). C'est la dimension « sacrificielle » de l'utilitarisme (Arnsperger, 2001): la société est considérée comme un corps social et l'individualité des hommes n'est pas prise au sérieux. En outre, l'objectif de maximisation de l'utilité se traduit logiquement par l'objectif d'égalisation des utilités marginales, c'est-à-dire par la réalisation de transferts de ressources de ceux qui les valorisent le moins (i.e. qui ont l'utilité marginale la plus faible) vers ceux qui les valorisent le plus (i.e. qui ont l'utilité marginale la plus élevée). Sen (1979) critique fortement cet objectif, car les seconds ne correspondent pas forcément aux plus nécessiteux. Avec un même niveau de ressource, une personne handicapée atteindra peut-être un niveau de satisfaction plus faible qu'une personne en pleine santé, mais cela ne devrait en aucun cas justifier un transfert de ressources de la première à la seconde.

\subsection{Le débat inspiré par la théorie de Rawls}

\subsubsection{Critiques de la théorie de Rawls}

La théorie de Rawls évite l'intuitionnisme en posant des règles de priorité entre principes. Le débat sur cette théorie a été et demeure intense. Un premier ensemble de 
critiques théoriques portent sur le principe de différence. Sen (1985) se demande si les gains - même minimes - du groupe social le plus désavantagé peuvent justifier n'importe quels sacrifices - même majeurs - des autres classes sociales. Cohen ${ }^{32}$ s'inquiète, au contraire, de l'étendue des inégalités socioéconomiques qu'autorise ce principe. Rawls y répond par des considérations pratiques : le cas édifié par la première critique n'a pas de portée empirique, quant à la seconde, elle oublie que les principes prioritaires d'égalité politique et d'égalité des chances réduiront fortement les inégalités auxquelles s'appliquent in fine le principe de différence. Une troisième critique concerne l'arbitraire de la définition du groupe social le plus défavorisé et de la construction de l'indice des biens sociaux premiers. Rawls (1997, p. 129) affirme que «toute procédure [d'application] est nécessairement ad hoc dans une certaine mesure » et que le problème est, dans les faits, plus simple qu'il n'y parait. Les membres du groupe social le plus désavantagé pourront être aisément identifiés, car ils auront une quantité plus faible de chaque bien premier, la construction de l'indice se limitera alors à la pondération des biens premiers pour ce groupe. Un second ensemble de critiques porte sur la place de la responsabilité et de la liberté dans la théorie de Rawls. Cohen (1989) fait remarquer que la focalisation sur les ressources assigne aux individus une responsabilité totale quant à leurs ambitions, alors que le principe de différence conduit à une responsabilité nulle quant à leurs choix ${ }^{33}$. Pour éviter ce «hiatus», la répartition devrait reposer intégralement sur le principe de la responsabilité morale: la justice devrait se focaliser uniquement sur les inégalités qui ne sont pas la conséquence des choix individuels. Sen $(1979,1985)$ critique la focalisation rawlsienne sur les ressources : il s'agit d'une erreur, car c'est aux fonctionnements ${ }^{34}$ et non aux biens auxquels les gens accordent de la valeur et, de plus, toutes les personnes n'ont pas la même capacité à convertir les biens en fonctionnements. Toutefois, une focalisation sur les fonctionnements risquerait de négliger la liberté et de conduire à une théorie perfectionniste. Puisque les gens accordent une valeur importante à la liberté de choisir la vie qu'ils souhaitent mener, la justice devrait se focaliser sur les ensembles de vecteurs de fonctionnements accessibles, autrement dit les capabilités. A nouveau, une fois les capabilités égalisées, les individus devraient être responsables des conséquences de leurs choix.

\subsubsection{Critiques des théories de l'égalité des chances}

Ce débat sur le traitement de la responsabilité et de la liberté est toujours d'actualité et conduit en retour à une première critique des théories de l'égalité des chances. L'idée qu'une personne qui épuise les chances initialement à sa disposition doit en subir

\footnotetext{
32 Cité par Arnsperger (2003).

33 Puisque l'utilité individuelle n'est pas considérée par la justice, chaque individu est intégralement responsable du degré de satisfaction atteint à l'aide des biens premiers à sa disposition, or ce degré dépend de ses ambitions personnelles. En revanche, l'individu n'est pas responsable de ses perspectives d'accès aux ressources, puisque ces dernières sont attachées aux positions sociales.

34 Rappelons que les fonctionnements sont «ce que les biens peuvent faire pour les gens », c'est-àdire toutes les possibilités d'être et de faire : être en bonne santé, bien logé, travailler, voyager, etc.
} 
toutes les conséquences parce qu'elle est moralement responsable de ses choix n'est pas convaincante du point de vue éthique (Fleurbaey, 2005, 2008). La justice sociale ne relève pas de la seule valeur de la responsabilité, mais aussi des valeurs de la liberté et de la solidarité. Il n'y a donc aucune raison pour que la sphère de responsabilité prise en compte par la justice, et définie par la société en référence à ces valeurs, soit équivalente à la sphère de la responsabilité morale ${ }^{35}$. D'une part, même si les chances sont égales ex ante, la justice est concernée par les inégalités et la pauvreté ex post 36 : c'est une évidence du point de vue de la solidarité, mais ce devrait l'être tout autant du point de vue de la liberté, car cette dernière est compromise lorsque l'autonomie de décision est rendue impossible par la pauvreté. D'autre part, il n'y a pas de raison morale profonde pour laquelle les individus devraient être attachés aux conséquences de leurs choix passés alors que leurs objectifs ont évolué : le droit à un nouveau départ constitue une liberté essentielle. Les chances devraient donc être égalisées tout au long de la vie et non pas à un "moment » de l'égalisation des chances, après lequel elles pourraient être dilapidées. Il n'existe d'ailleurs aucun argument justifiant la référence usuelle à l'entrée dans la vie adulte comme moment de l'égalisation des chances (Chambers, 2006). En somme, le problème de la justice n'est pas de traquer les choix passés erronés pour identifier les inégalités équitables, mais d'assurer à tout moment une égalité dans la capacité de chacun à décider des orientations de sa vie future (Fleurbaey, 2008). Une seconde critique porte sur la cohérence interne des théories de l'égalité des chances. Ces théories ne font pas appel à un principe d'égalisation, mais à deux principes distincts: les principes de compensation des circonstances et de récompense libérale (Fleurbaey, 2008). Le premier requiert que deux individus effectuant des choix similaires atteignent un niveau de bien-être égal, c'est-à-dire que les inégalités associées à des circonstances non choisies soient intégralement compensées. Le second impose le "laisser faire » lorsque deux individus font face aux mêmes circonstances, de manière à ce qu'ils bénéficient intégralement des récompenses légitimes associées à leurs choix. Or, ces deux principes ne peuvent être satisfaits conjointement ${ }^{37}$. Enfin, une dernière critique importante porte sur l'intérêt respectif des deux conceptions principales du choix. Selon Fleurbaey (2002), la distinction entre préférences et circonstances est la seule à disposer d'un contenu précis. En effet, identifier les choix véritables requiert de définir le libre arbitre; il s'agit d'un problème d'ordre métaphysique, impossible à résoudre en pratique.

Il est manifeste que le débat philosophique n'est pas tranché et ses termes demanderaient à être longuement développés. Bien que le choix de la conception de la justice sur laquelle adosser l'action publique constitue l'une des questions des plus ardues qui soient, nous pensons que ce débat mérite d'être posé dans le cadre du fonctionnement des institutions démocratiques, afin de mieux raisonner et justifier l'action publique. Pour ce qui a trait à la politique rurale, nous pouvons retenir que la pertinence de ses orientations, sous l'angle du débat philosophique contemporain, est sérieusement mise en doute. D'une part, leurs fondements

\footnotetext{
35 A ce titre, le «hiatus » rawlsien évoqué plus haut n'en est pas un.

36 Ex ante et ex post se référant aux situations avant et après l'exercice du choix.

37 Voir les démonstrations de Fleurbaey (2008) à partir d'exemples et de manière formelle.
} 
intuitionnistes sont rejetés par de nombreuses théories philosophiques; or, nous avons vu précédemment que ces dernières conduiraient à des orientations sensiblement différentes. D'autre part, quand bien même l'intuitionnisme devait être reconnu comme un horizon indépassable, il reste que les objectifs assignés à la politique rurale ne s'appuient pas sur une véritable conception intuitionniste de la justice, dont la cohérence interne et la pertinence seraient philosophiquement argumentées.

\section{Conclusion}

Nous avons fait appel à la philosophie morale et politique pour constituer le référentiel d'évaluation de la pertinence des objectifs de la politique rurale française. A cet égard, notre principale conclusion est la suivante: leur pertinence est incertaine, car ils reposent sur des fondements philosophiques implicites fragiles et contestables. Les objectifs énoncés sont multiples et potentiellement contradictoires: assurer la valorisation économique, sociale et environnementale des territoires ruraux; la croissance économique nationale; l'augmentation générale de l'emploi; la cohésion sociale et territoriale; la gestion durable du patrimoine culturel, etc. Aucun critère n'est proposé pour définir les termes d'un arbitrage entre ces derniers et les acteurs impliqués concrètement dans sa négociation doivent s'en remettre à leurs intuitions ou à leurs intérêts. Ces caractéristiques sont précisément celles d'une conception intuitionniste de la justice. Or, les principales conceptions de la justice se rattachant aux doctrines philosophiques perfectionniste, utilitariste, rawlsienne ou de l'égalité des chances, en particulier, réfutent la doctrine intuitionniste et définissent clairement un objectif supérieur ou une hiérarchie d'objectifs pour orienter l'action publique. Nous avons vu, en outre, que si de telles conceptions étaient retenues par l'Etat, elles conduiraient vraisemblablement à des politiques rurales sensiblement différentes. De plus, quand bien même la doctrine intuitionniste devrait être acceptée, il reste que le choix des objectifs de la politique rurale ne s'appuie sur aucune argumentation philosophique.

Au-delà de l'analyse de la pertinence d'une politique particulière, cette réflexion nous conduit à penser que le problème fondamental est celui de l'adoption par l'Etat d'une conception de la justice sur laquelle fonder son action. C'est évidemment dans le cadre du fonctionnement des institutions démocratiques que le choix d'une conception de la justice pourrait être débattu. La légitimité de l'action publique, dans son rapport à la demande sociale de transparence et d'impartialité, s'en trouverait certainement augmentée. Cet effort d'explicitation de la conception de la justice sous-jacente à l'action publique serait une marque de progression vers l'idéal rawlsien d'une société bien ordonnée, où chacun accepte et sait que les autres acceptent les mêmes principes de la justice, et où les politiques contribuent à leur mise en œuvre.

Ce parti pris de l'ensemble de notre analyse - que la progression vers cet idéal est souhaitable - semble toutefois mis à l'épreuve par les faits. Cet idéal est intimement associé à celui d'une société s'organisant sur la base d'un contrat social établi entre citoyens au niveau national. Or, dans la société française d'aujourd'hui, les mouvements de déconcentration administrative, de décentralisation politique et, plus généralement, de territorialisation de l'action publique tendent à donner une importance grandissante 
à la citoyenneté locale et à la «demande sociale locale». Les pratiques usuelles d'évaluation sont d'ailleurs cohérentes avec ces évolutions, considérant que les objectifs d'une politique sont pertinents s'ils convergent avec les «problèmes socioéconomiques» généralement identifiés localement. Cet enracinement territorial de l'action publique possède des propriétés favorables à la recherche de modalités d'action adaptées au contexte, mais pose question sur le plan de la définition des finalités politiques. Doit-on abandonner l'idée de définir la justice sociale sur la base d'un contrat social national et envisager l'échelon local comme adéquat pour «faire société » de ce point de vue ? Ou encore, est-il possible de définir la justice sociale à plusieurs échelons géographiques simultanément? Dans le cas contraire, si le contrat social national et la société bien ordonnée sont effectivement retenus comme modèles, peuton vraiment transférer le pouvoir aux territoires et, dans le même temps, assurer la justice à l'échelle nationale ? Ces questions cruciales méritent également d'être posées dans le débat public.

\section{Bibliographie}

Arneson R.J. (1989) Equality and equal opportunity for welfare, Philosophical studies 56(1), 77-93.

Arnsperger C. (2003) Peut-il exister une critique sociale rawlsienne?, Mouvements 27/28, $165-170$.

Arnsperger C. (2001) Entre impartialité, horizons de sens et précarité existentielle, les fondements de l'éthique économique et sociale, in: Trois essais sur l'éthique économique et sociale, Arnsperger C., Larrère C. et Ladrière J., Versailles, INRA éditions, 19-83.

Berriet-Solliec M., Schmitt B., Trouvé A. et Aubert F. (2009) Second pilier de la PAC et développement rural: le RDR est-il vraiment rural?, in : Politiques agricoles et territoires, Aubert F., Piveteau V. et Schmitt B. (coord.), Versailles, Editions Quae, chapitre 7.

Callois J.-M. (2006) Quality labels and rural development: A new economic geography approach, Cabiers d'économie et de sociologie rurales 78, 31-51.

Chabé-Ferret S. (2008) Evaluer l'impact des politiques publiques: caractérisation des enjeux et exemples de politiques agricoles et forestières, Thèse de doctorat en sciences économiques, Clermont-Ferrand, Université d'Auvergne - Clermont I, 25 juin, $262 \mathrm{p}$.

Chambers C. (2006) Each outcome is another opportunity, Working paper Series $\mathrm{n}^{\circ}$ SJ001, Oxford, Centre for the Study of Social Justice.

Charlot S. (2001) Politiques publiques et délocalisation des activités vers les espaces ruraux: une analyse théorique, Cabiers d'économie et sociologie rurales 58-59, 170-192. 
Cohen G.A. (1989) On the currency of egalitarian justice, Ethic 99, 906-944.

Conseil de l'Union européenne (2006) Décision relative aux orientations stratégiques communautaires en matière de cohésion, 2006/702/CE, JOUE du 21 octobre, $291 / 11$ à 291/32.

Commission européenne, Fonds structurels communautaires (1999) Glossaire de 300 concepts et termes techniques, volume 6 de la collection MEANS : Evaluer les programmes socio-économiques, Luxembourg, Office des publications des Communautés européennes.

Daniel K., Kilkenny M. (2009) Agricultural subsidies and rural development, Journal of Agricultural Economics 60(3), 504-529.

Dworkin R. (2000) Sovereign Virtue, The Theory and Practice of Equality, Cambridge (MA), Harvard University Press.

Dworkin R. (1981) What is equality?, Part 2 : Equality of resources, Philosophy and Public Affairs 10(4), 283-345.

Fleurbaey M. (2008) Fairness, Responsibility, and Welfare, Oxford, Oxford University Press.

Fleurbaey M. (2005) Freedom with forgiveness, Politics, philosophy $\mathcal{E}$ economics 4(1), 29-67.

Fleurbaey M. (2002) Equality of resources revisited, Ethics 113(1), 82-105.

Guérin M. (2006) Evolution des objectifs et des principes d'intervention des politiques de développement rural sur le long-terme, in: Politiques de développement rural : enjeux, modalités et stratégies, Guérin $M$. (éd.), Ingénieries no spécial FEADER, 27-38.

Guichard O. (1965) Aménager la France, Paris, Laffont-Gonthier.

Konow J. (2003) Which is the fairest one of all? A positive analysis of justice theories, Journal of Economic Literature 41(4), 1188-1239.

Larrère C. (2006) Ethiques de l'environnement, Multitudes 24, 75-84.

Lofredi P. (2007) Evaluation économétrique des effets de traitement et programmes de développement à ciblage géographique. Le cas des interventions économiques en faveur du développement rural, Doctorat en sciences économiques de l'Université de Bourgogne, UMR CESAER (INRA-ENESAD), Dijon.

Lorenceau A. (2009) L'impact d'exonérations fiscales sur la création d'entreprises et l'emploi en France rurale: une approche par discontinuité de la régression, Communication aux Journées économie et espace, Dijon, 29 et 30 juin.

Mac Intyre A. (1981) After Virtue, Notre Dame, University of Notre Dame Press.

Ministère de l'agriculture et de la pêche (2007) Plan stratégique national de développement rural 2007-2013, Paris, 16 avril.

Moulignier A., Mondot R. (2005) Le Fonds européen agricole pour le développement rural : un nouveau cadre financier pour une politique rénovée, in : Politiques de 
développement rural : enjeux, modalités et stratégies, Guérin M. (éd.), Ingénieries $\mathrm{n}^{\circ}$ spécial FEADER, 11-26.

Nozick R. (1974) Anarchy, State and Utopia, New York, Basic Books.

Perrin D., Mallet J., Brun G., Guérin M., Ruas J.-F., Templé P. et Teurkauff M.-C. (2003) Les politiques de développement rural, Commissariat général du plan, Paris, La Documentation française.

Rawls J. (1997) Théorie de la justice (trad. A Theory of Justice, 1971), Paris, Editions du Seuil.

Roemer J.E. (1998) Equality of Opportunity, Cambridge (MA), Havard University Press.

Sen A. (1985) Commodities and Capabilities. Professor Dr. P. Hennipman Lectures in Economics: Theory, Institutions, Policy, Amsterdam, North-Holland, volume 7.

Sen A. (1979) Equality of What? in: The Tanner Lecture On Human Values, delivered at Stanford University, May 22.

Trouvé A., Berriet-Solliec M. (2008) 2nd pilier de la Politique agricole commune et régionalisation : vers plus de cohésion? Revue d'Economie Régionale et Urbaine 1, 87-108.

Walzer M. (1983) Spheres of Justice, New York, Basic Books. 
